\title{
Effect of bitter tastants on human bronchi
}

\section{To the Editor:}

We read with interest the report of the effects of bitter tastants on airway smooth muscle by Deshpande et al. ${ }^{1}$ and the related News and Views article by Sanderson and Madison ${ }^{2}$. Deshpande et al. ${ }^{1}$ report a previously undescribed modulator of airway tone with a unique mode of relaxation in airway smooth muscle that may prove to be clinically significant. We attempted to reproduce the data presented using second-order human bronchi obtained from people with lung cancer after surgical resection. We set rings ( $n=24$ from nine subjects) at a passive tension of $2 \mathrm{~g}$ and contracted them with $1 \mathrm{mM}$ methacholine or $1 \mathrm{mM}$ acetylcholine. We did control relaxations using $10 \mu \mathrm{M}$ isoprenaline $(n=9)$. In contrast to the report by Deshpande et al. ${ }^{1}$, isoprenaline induced rapid $(7.9 \pm 5.9 \mathrm{~min})$ and potent relaxation of bronchi constricted with acetylcholine ( $145 \pm 39 \%$ inhibition of maximum, $n=3)$ and methacholine (103 $\pm 49 \%$ inhibition of maximum, $n=6$ ) (Fig. 1a). Their claim that bitter taste receptor agonists have three times the efficacy of $\beta$-agonists ${ }^{1}$ may result from the poor performance of isoprenaline in their report. Our own experience and that reported in the literature $^{3,4}$ is that isoprenaline is a potent and highly efficacious bronchodilator of human airway smooth muscle. We repeated the experiments reported by Deshpande et al. ${ }^{1}$ using three of the bitter tastants as bronchodilators in bronchi constricted with $1 \mathrm{mM}$ methacholine. Saccharin $(n=5)$ produced no response up to concentrations of $3 \mathrm{mM}$ (Fig. 1b). Quinine ( $1 \mathrm{mM} ; n=5)$ and $1-3 \mathrm{mM}$ chloroquine $(n=5)$ induced relaxation of bronchi (Fig. 1b). At these concentrations, we found that the average time for relaxation to baseline was $23 \pm 6 \mathrm{~min}$ for quinine and 34 \pm 16 min for chloroquine (Fig. 1b). After a mean washout time of $37 \pm$ $10 \mathrm{~min}$, the contractile response to methacholine was reduced $(15 \pm 19 \%$ and $27 \pm 15 \%$ of the rings that were pre-exposed to quinine and chloroquine, respectively (Fig. 1b)). Our inability to reverse the effects of bitter tastants in the human bronchial preparation used in our study stands in contrast to the report by Deshpande et al. ${ }^{1}$, in which chloroquine mediated-relaxation was fully reversible in mouse tracheal rings.

We interpret these findings as showing that, at very high concentrations, bitter tastants may indeed relax smooth muscle; however, our inability to reverse the effect in washout suggests either irreversible inhibition of contraction or cell injury. Some of the differences between our results may be explained by the use of second-order bronchi in our studies as compared with the fourth-order bronchi in the study by Deshpande et al. ${ }^{1}$. Different pharmacological classes of agonists have differential effects depending on airway diameter ${ }^{5}$. In asthma, large airways are thought to be the main contributors to airflow obstruction, whereas in chronic obstructive pulmonary disease, smaller airway constriction has a greater role in the pathophysiology. The localization and function of bitter taste receptors within human airways needs clarification if we are to understand the possible roles of TAS2R agonists as bronchodilators.

Note: Supplementary information is available on the Nature Medicine website.
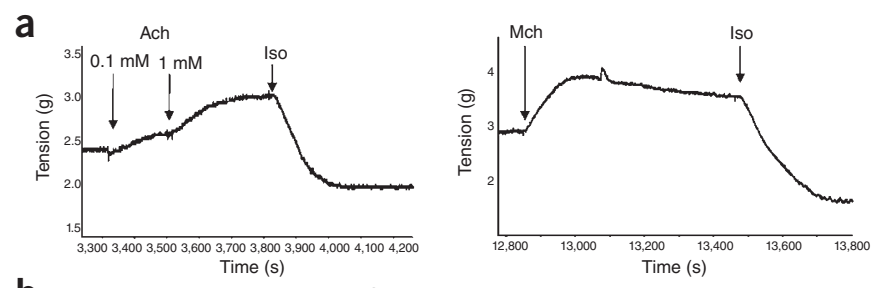

b
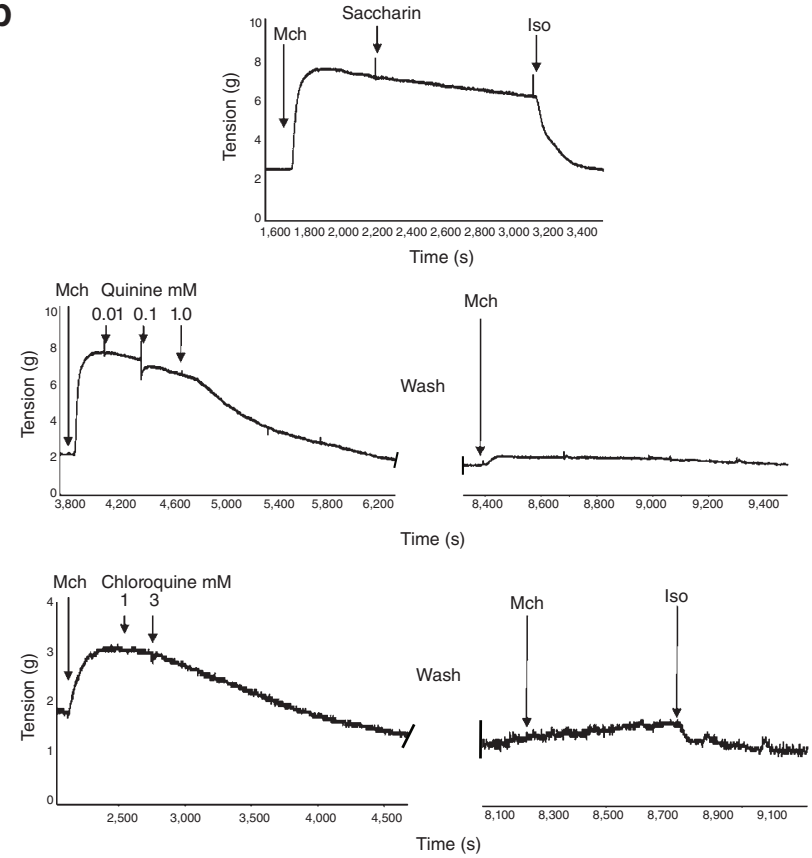

Figure 1 Relaxation of human bronchi to isoprenaline and bitter tastants. (a) Representative traces from two bronchial rings contracted with $1 \mathrm{mM}$ acetylcholine (Ach) or $1 \mathrm{mM}$ methacholine (Mch) before relaxation to $10 \mu \mathrm{M}$ isoprenaline (Iso). (b) Representative traces showing effects of $1 \mathrm{mM}$ saccharin, 0.01-1 mM quinine and 1-3 mM chloroquine on bronchi precontracted with $1 \mathrm{mM}$ methacholine. The break in the lower two traces represents a 30-min washout before repeat contraction with $1 \mathrm{mM}$ methacholine.

COMPETING FINANCIAL INTERESTS

The authors declare no competing financial interests.

Alyn H Morice ${ }^{1}$, Robert T Bennett ${ }^{1}$, Mubarak A Chaudhry ${ }^{2}$, Michael E Cowen ${ }^{2}$, Steven C Griffin ${ }^{2}$ \& Mahmoud Loubani ${ }^{2}$

${ }^{1}$ Cardiovascular and Respiratory Studies, University of Hull, Hull York Medical School, Hull, United Kingdom. ${ }^{2}$ Centre for Cardiology and Cardiothoracic Surgery, Castle Hill Hospital, Cottingham, United Kingdom. e-mail:a.h.morice@hull.ac.uk

1. Deshpande, D.A. et al. Nat. Med. 16, 1299-1304 (2010)

2. Sanderson, M.J. \& Madison, J.M. Nat. Med. 16, 1190-1191 (2010).

3. Naline, E. et al. Eur. Respir. J. 7, 914-920 (1994).

4. Yamagata, S. et al. Pulm. Pharmacol. Ther. 13, 25-29 (2000).

5. Ohrui, T. et al. Am. Rev. Respir. Dis. 146, 88-91 (1992). 